\title{
New Results on Stochastic Comparison of Series and Parallel Systems Comprising Heterogeneous Generalized Modified Weibull Components
}

\author{
Esmaeil Bashkar \\ Velayat University \\ Received: 3/25/2020 Approved: 10/30/2020
}

\begin{abstract}
In this work, we study various stochastic orderings of the smallest and largest order statistics arising from independent heterogeneous generalized modified Weibull (GMW) random variables. We also conduct stochastic comparison on the extreme order statistics from GMW samples with Archimedean copulas. The results established in this paper strengthen and generalize those known in the Balakrishnan et al. (2018).
\end{abstract}

Keywords. Majorization; order statistics; series and parallel systems; stochastic orders; generalized modified Weibull distribution.

MSC 2010: 60E15, 60E10.

\section{Introduction}

Series and parallel systems are two basic systems which play important roles in various applications in reliability engineering. An $n$-component system with series (parallel) structure fails (works) if at least one of the components of the system fails (works). Let $X_{1}, X_{2}, \ldots, X_{n}$ denote the lifetimes of $n$ components that can be used to built up an $n$ component system. If $X_{1: n} \leq \ldots \leq X_{n: n}$ denote the ordered lifetimes of the components then it is 
known that $X_{1: n}$ and $X_{n: n}$ correspond to the lifetimes of series and parallel systems, respectively. Reliability and stochastic properties of series and parallel systems have been considered by various researchers under different scenarios. For example, stochastic comparisons of the lifetimes of series and parallel systems, in the case of heterogeneous component lifetimes with Weibull distributions, are considered in Khaledi and Kochar (2006), Fang and Zhang (2013), Torrado (2015), Li and Li (2015), Torrado and Kochar (2015) and Fang and Balakrishnan (2016). Attempts have also been made by Fang and Zhang (2015) and Kundu and Chowdhury (2016) in the case of heterogeneous components with exponentiated Weibull (EW) distributions, and by Balakrishnan et al. (2014) in the case of heterogeneous components with generalized exponential (GE) distributions. For a recent review on the topic one can refer to Balakrishnan and Zhao (2013).

The aim of the present note is to compare the lifetimes of series and parallel systems with heterogeneous components where the component lifetimes are distributed as a four-parameter generalized modified Weibull (GMW) distribution with cumulative distribution function (cdf)

$$
F(x)=\left(1-\exp \left(-\alpha x^{\gamma} \exp (\lambda x)\right)^{\beta}\right.
$$

where $\alpha>0$ is a scale parameter, and $\gamma>0$ and $\beta>0$ are shape parameters. The parameter $\lambda$ works as a factor of fragility in the survival of an individual as time increases. This model which has recently been studied by Carrasco et al. (2008), has both monotone and nonmonotone hazard rates. If a random variable $X$ has GMW distribution in (1), then we write $X \sim \operatorname{GMW}(\alpha, \gamma, \lambda, \beta)$. For $\lambda=0$ and $\beta=1$, we obtain the Weibull distribution and we have modified Weibull distributions when $\beta=1$. For $\lambda=0$ the model (1) reduces to the EW distribution constructed by Mudholkar and Srivastava (1993). Our established results extend some known results dealing with Weibull and EW distributions. Furthermore, the results obtained here strengthen and generalize those known in the Balakrishnan et al. (2018).

The rest of the paper is organized as follows: In Section 2, we give the required definitions and some useful lemmas which are used throughout the paper. In Section 3, we discuss stochastic comparisons of the lifetimes of series and parallel systems with independent heterogeneous GMW components in terms of the usual stochastic order, hazard rate order and the reversed hazard rate order. Section 4 discusses some results on series systems with dependent components where the structure of dependency is expressed according to the Archimedean copula. Throughout this paper, we use the no- 
tations $\mathbb{R}=(-\infty,+\infty), \mathbb{R}_{++}=(0,+\infty), \mathbb{D}_{+}=\left\{\left(x_{1}, x_{2}, \ldots, x_{n}\right): x_{1} \geq x_{2} \geq\right.$ $\left.\cdots \geq x_{n}>0\right\}, \mathbb{E}_{+}=\left\{\left(x_{1}, x_{2}, \ldots, x_{n}\right): 0<x_{1} \leq x_{2} \leq \cdots \leq x_{n}\right\}$ and use the term 'increasing' for nondecreasing and 'decreasing' for nonincreasing.

\section{Preliminaries}

In this section, we review some definitions and well-known notions of stochastic orders and majorization concepts and lemmas that are useful for proving our main results. Let $X$ and $Y$ be two non-negative continuous random variables with distribution functions $F$ and $G$, density functions $f$ and $g$, and the survival functions $\bar{F}=1-F$ and $\bar{G}=1-G$, respectively. Denote also the reverse hazard rate functions $r_{F}=\frac{f}{F}$ and $r_{F}=\frac{f}{F}$, hazard rate functions of $X$ and $Y$ by $h_{F}=\frac{f}{F}$ and $h_{G}=\frac{g}{G}$, respectively. The following definition introduces some well-known orders that compare the magnitude of two random variables.

Definition 1. $X$ is said to be smaller than $Y$ in the

(i) stochastic order, denoted by $X \leq_{\text {st }} Y$, if $\bar{F}(x) \leq \bar{G}(x)$ for all $x$;

(ii) hazard rate order, denoted by $X \leq_{\mathrm{hr}} Y$, if $h_{F}(x) \geq h_{G}(x)$ for all $x$.

(iii) reversed hazard rate order, denoted by $X \leq_{r h} Y$, if $r_{F}(x) \leq r_{G}(x)$ for all $x$.

Note that the hazard rate order, and the reversed hazard rate order implies the usual stochastic order. For more on these stochastic orders one may refer to Shaked and Shanthikumar (2007) and Li and Li (2013).

A real function $\phi$ is said to be $n$-monotone on $(a, b) \subseteq(-\infty,+\infty)$ if $(-1)^{n-2} \phi^{(n-2)}$ is decreasing and convex in $(a, b)$ and $(-1)^{k} \phi^{(k)}(x) \geq 0$ for all $x \in(a, b), k=0,1, \ldots, n-2$, where $\phi^{(i)}($.$) is the i$ th derivative of $\phi($.$) . For$ a $n$-monotone $(n \geq 2)$ function $\phi:[0,+\infty) \longrightarrow[0,1]$ with $\phi(0)=1$ and $\lim _{x \rightarrow+\infty} \phi(x)=0$, let $\psi=\phi^{-1}$ be the pseudo-inverse, then

$$
C_{\phi}\left(u_{1}, \ldots, u_{n}\right)=\phi\left(\psi\left(u_{1}\right)+\ldots+\psi\left(u_{n}\right)\right), \quad \text { for all } u_{i} \in[0,1], i=1, \ldots, n,
$$

is called an Archimedean copula with the generator $\phi$. Archimedean copulas cover a wide range of dependence structures including the independence copula with the generator $\phi(t)=e^{-t}$. For more detail on Archimedean copula, see, McNeil and Neslehova (2009) and Nelsen (2006). 
The notion of majorization is essential for the understanding of the stochastic inequalities for comparing order statistics. In the sequel, we use the notation $x_{(1)} \leq x_{(2)} \leq \ldots \leq x_{(n)}$ to denote the increasing arrangement of the components of the vector $\boldsymbol{x}=\left(x_{1}, \ldots, x_{n}\right)$.

Definition 2. The vector $\boldsymbol{x}$ is said to be

(i) weakly submajorized by the vector $\boldsymbol{y}$ (denoted by $\boldsymbol{x} \preceq_{\mathrm{w}} \boldsymbol{y}$ ) if $\sum_{i=j}^{n} x_{(i)} \leq$ $\sum_{i=j}^{n} y_{(i)}$ for all $j=1, \ldots, n$,

(ii) weakly supermajorized by the vector $\boldsymbol{y}$ (denoted by $\boldsymbol{x} \stackrel{\mathrm{w}}{\preceq} \boldsymbol{y}$ ) if $\sum_{i=1}^{j} x_{(i)} \geq$ $\sum_{i=1}^{j} y_{(i)}$ for all $j=1, \ldots, n$,

(iii) majorized by the vector $\boldsymbol{y}$ (denoted by $\boldsymbol{x} \preceq \boldsymbol{m}$ ) if $\sum_{i=1}^{n} x_{i}=\sum_{i=1}^{n} y_{i}$ and $\sum_{i=1}^{j} x_{(i)} \geq \sum_{i=1}^{j} y_{(i)}$ for all $j=1, \ldots, n-1$.

Bon and Pǎltǎnea (1999) introduced the order of $p$-larger.

Definition 3. A vector $\boldsymbol{x}$ in $\mathbb{R}_{+}^{n}$ is said to be $p$-larger than another vector $\boldsymbol{y}$ in $\mathbb{R}_{+}^{n}$ (denoted by $\boldsymbol{x} \stackrel{\mathrm{p}}{\succeq} \boldsymbol{y}$ ) if

$$
\prod_{i=1}^{j} x_{(i)} \leq \prod_{i=1}^{j} y_{(i)}, \quad \text { for } j=1, \ldots, n .
$$

Definition 4. A real valued function $\varphi$ defined on a set $A \subseteq \mathbb{R}^{n}$ is said to be Schur-convex (Schur-concave) on $A$ if

$$
\boldsymbol{x} \preceq \boldsymbol{y} \quad \text { on } \quad A \Longrightarrow \varphi(\boldsymbol{x}) \leq(\geq) \varphi(\boldsymbol{y}) .
$$

For more details on the above partial orders of real vectors, Schur-convexity and Schur-concavity we refer readers to Marshall et al. (2011). Let us introduce the following lemmas which will be used in the next sections to prove the results.

Lemma 1 (Marshall et al. (2011), Theorem 3.A.4). Suppose $\mathbb{I} \subset \mathbb{R}$ is an open interval and $\Phi: \mathbb{I}^{n} \longrightarrow \mathbb{R}_{+}$is continuously differentiable. Necessary and sufficient conditions for $\Phi$ to be Schur-convex (Schur-concave) on $\mathbb{I}^{n}$ are

(i) $\Phi$ is symmetric on $\mathbb{I}^{n}$, 
(ii) for $i \neq j$ and all $z \in \mathbb{I}^{n}$,

$$
\left(z_{i}-z_{j}\right)\left(\frac{\partial \Phi(z)}{\partial z_{i}}-\frac{\partial \Phi(z)}{\partial z_{j}}\right) \geq(\leq) 0
$$

where $\frac{\partial \Phi(z)}{\partial z_{i}}$ denotes the partial derivative of $\Phi$ with respect to its $i$-th argument.

Lemma 2 (Marshall et al. (2011), Theorem 3.A.8). For a function $l$ on $A \in \mathbb{R}^{n}, \boldsymbol{x} \preceq_{\mathrm{w}}(\stackrel{\mathrm{w}}{\preceq}) \boldsymbol{y}$ implies $l(\boldsymbol{x}) \leq l(\boldsymbol{y})$ if and only if it is increasing (decreasing) and Schur-convex on $A$.

Lemma 3. The function $g:(0, \infty) \times(0,1) \longrightarrow(0, \infty)$ defined as

$$
g(\beta, x)=\frac{\beta x^{\beta-1}}{1-x^{\beta}}
$$

is a decreasing function of $\beta$.

Proof. It is easy to see that $g(\beta, x)=\frac{1}{x} \frac{\beta x^{\beta}}{1-x^{\beta}}$ and

$$
\begin{gathered}
\frac{\partial g(\beta, x)}{\partial \beta} \stackrel{\operatorname{sgn}}{=}\left(x^{\beta}+x^{\beta} \log (x) \beta\right) \times\left(1-x^{\beta}\right)+\beta x^{2 \beta} \log (x)= \\
x^{\beta}\left(1+\beta \log (x)-x^{\beta}\right)=: f(\beta) .
\end{gathered}
$$

Then, it follows that

$$
\frac{\partial f(\beta)}{\partial \beta}=\log (x)\left(1-x^{\beta}\right)<0
$$

Therefore, $f(\beta)<f(0)=0$, and so $\frac{\partial g(\beta, x)}{\partial \beta}<0$. The assertion then follows.

\section{Main Results}

\subsection{Independent Random Variables}

In this section, we provide some new comparison results on the lifetimes of series and parallel systems arising from independent heterogeneous GMW random variables. 
The following results discuss the variability ordering of lifetimes of parallel systems with independent heterogeneous GMW components in terms of the reversed hazard rate order.

Theorem 1. Let $X_{1}, \ldots, X_{n}$ be a set of independent random variables with $X_{i} \sim \operatorname{GMW}\left(\alpha_{i}, \gamma, \lambda, \beta\right)$ ، $i=1, \ldots, n$. Further، let $X_{1}^{*}, \ldots, X_{n}^{*}$ be another set of independent random variables with $X_{i}^{*} \sim \operatorname{GMW}\left(\alpha_{i}^{*}, \gamma, \lambda, \beta\right)$ ، $i=1, \ldots, n$. If $\left(\alpha_{1}, \ldots, \alpha_{n}\right) \stackrel{\mathrm{w}}{\preceq}\left(\alpha_{1}^{*}, \ldots, \alpha_{n}^{*}\right)$, then $X_{n: n} \leq_{\mathrm{rh}} X_{n: n}^{*}$.

Proof. Let us consider a fixed $x>0$, then the reversed hazard rate of $X_{n: n}$ is given by

$$
\tilde{r}_{X_{n: n}}(x)=\frac{\beta(\lambda x+\gamma)}{x} \sum_{i=1}^{n} \frac{u_{i}}{e^{u_{i}}-1}
$$

where $u_{i}=\alpha_{i} x^{\gamma} e^{\lambda x}$. From Lemma 2, the proof follows if we prove that, for each $x>0, \tilde{r}_{X_{n: n}}(x)$ is Schur-convex and decreasing in $\alpha_{i}$ 's. For the proof of the first part, see the work of Balakrishnan et al. (2018). The proof of the second part is easy. Thus, it is omitted for the sake of conciseness.

Note that $\left(\alpha_{1}, \ldots, \alpha_{n}\right) \stackrel{\mathrm{m}}{\preceq}\left(\alpha_{1}^{*}, \ldots, \alpha_{n}^{*}\right)$ implies $\left(\alpha_{1}, \ldots, \alpha_{n}\right) \stackrel{\mathrm{w}}{\preceq}\left(\alpha_{1}^{*}, \ldots, \alpha_{n}^{*}\right)$, Theorem 1 substantially improves Theorem 1 of Balakrishnan et al. (2018).

Example 1. Let $\left(X_{1}, X_{2}, X_{3}\right)\left(\left(X_{1}^{*}, X_{2}^{*}, X_{3}^{*}\right)\right)$ be a vector of independent heterogeneous GMW random variables. Set $\gamma=2, \lambda=1.2, \beta=0.5,\left(\alpha_{1}, \alpha_{2}, \alpha_{3}\right)=$ $(0.1,4,6)$ and $\left(\alpha_{1}^{*}, \alpha_{2}^{*}, \alpha_{3}^{*}\right)=(0.1,1,8)$. Obviously $\left(\alpha_{1}, \alpha_{2}, \alpha_{3}\right) \stackrel{\mathrm{w}}{\preceq}\left(\alpha_{1}^{*}, \alpha_{2}^{*}, \alpha_{3}^{*}\right)$, and $X_{3: 3}$ and $X_{3: 3}^{*}$ are ordered in the reversed hazard rate order, as can be seen in Fig. 1.

We now generalize Theorem 2 to a wider range of the shape parameters as follows.

Theorem 2. Let the conditions of Theorem 1 be met. Then, for any $\gamma, \lambda, \beta>$ 0 , if $\alpha_{i} \leq \alpha_{i}^{*}, i=1, \ldots, n$, we have $X_{n: n} \geq_{\mathrm{rh}} X_{n: n}^{*}$.

Proof. Using the definition of the reversed hazard rate order and the fact that $\tilde{r}_{X_{n: n}}(x)$ is decreasing in each $\alpha_{i}$, the required result follows readily.

Theorem 3. For $i=1, \ldots, n$, let $X_{i}$ and $X_{i}^{*}$ be two sets of mutually independent random variables with $X_{i} \sim \operatorname{GMW}\left(\alpha_{i}, \gamma, \lambda, \beta_{i}\right)$ and $X_{i}^{*} \sim \operatorname{GMW}\left(\alpha_{i}^{*}, \gamma, \lambda, \beta_{i}\right)$. Further, suppose that $\boldsymbol{\beta} \in \mathbb{E}_{+}$and $\boldsymbol{\alpha}, \boldsymbol{\alpha}^{*} \in \mathbb{D}_{+}$. Then for all $\gamma, \lambda>0$, $\left(\alpha_{1}, \ldots, \alpha_{n}\right) \stackrel{\mathrm{w}}{\preceq}\left(\alpha_{1}^{*}, \ldots, \alpha_{n}^{*}\right)$ implies $X_{n: n} \leq_{\mathrm{rh}} X_{n: n}^{*}$ 


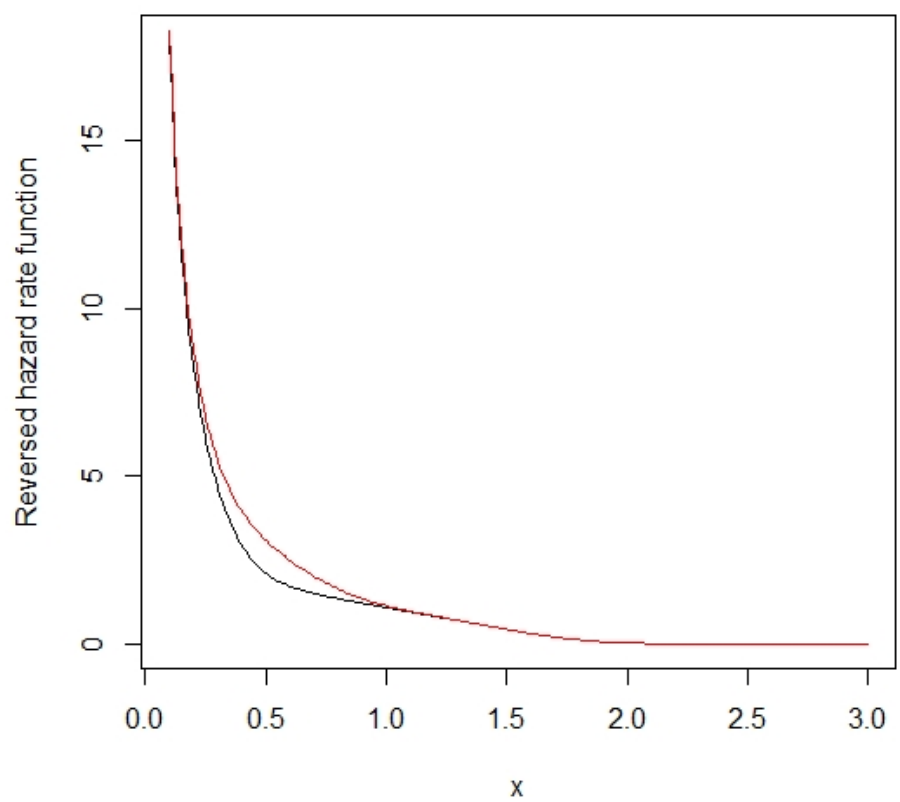

Figure 1. Plot of the reversed hazard rate functions of $X_{3: 3}$ (black line) and $X_{3: 3}^{*}$ (red line)

Proof. The reversed hazard rate functions of $X_{n: n}$ is given by

$$
\tilde{r}_{X_{n: n}}(x)=\frac{(\lambda x+\gamma)}{x} \sum_{i=1}^{n} \frac{\beta_{i} \alpha_{i} x^{\gamma} e^{\lambda x}}{e^{\alpha_{i} x^{\gamma} e^{\lambda x}}-1} .
$$

Let $g_{i}(y)=\frac{\beta_{i} y x^{\gamma} e^{\lambda x}}{e^{y x^{\gamma} e^{\lambda x}}-1}=\beta_{i} h\left(y x^{\gamma} e^{\lambda x}\right)$, where, by Lemma 8 of Balakrishnan et al. (2018), $h(y)=\frac{y}{e^{y}-1}$ is convex and decreasing in $y>0$, which implies that for any two real numbers $a \geq b, g^{\prime}{ }_{i}(a) \geq g^{\prime}{ }_{i}(b)$. So, by Proposition H.2 of Marshall et al. (2011), $\tilde{r}_{X_{n: n}}(x)$ is Schur convex. Thus, the result follows from Lemma 8 of Balakrishnan et al. (2018) and lemma 2.

Theorem 4. For $i=1, \ldots, n$, let $X_{i}$ and $X_{i}^{*}$ be two sets of mutually independent random variables with $X_{i} \sim \operatorname{GMW}\left(\alpha_{i}, \gamma, \lambda, \beta_{i}\right)$ and $X_{i}^{*} \sim \operatorname{GMW}\left(\alpha_{i}^{*}, \gamma, \lambda, \beta_{i}^{*}\right)$. 
Then, for any $\gamma, \lambda>0$, if $\alpha_{i} \geq \alpha_{i}^{*}$ and $\beta_{i} \leq \beta_{i}^{*}, i=1, \ldots, n$, we have $X_{n: n} \leq_{\mathrm{rh}} X_{n: n}^{*}$.

Proof. Using the definition of the reversed hazard rate order and the fact that $\tilde{r}_{X_{n: n}}(x)$ is decreasing in each $\alpha_{i}$ and increasing in each $\beta_{i}$, the required result follows readily.

Theorem 5. For $i=1, \ldots, n$, let $X_{i}$ and $X_{i}^{*}$ be two sets of mutually independent random variables with $X_{i} \sim \operatorname{GMW}\left(\alpha_{i}, \gamma, \lambda, \beta_{i}\right)$ and $X_{i}^{*} \sim \operatorname{GMW}\left(\alpha_{i}, \gamma, \lambda, \beta_{i}^{*}\right)$. If $\left(\beta_{1}, \ldots, \beta_{n}\right) \stackrel{\mathrm{m}}{\preceq}\left(\beta_{1}^{*}, \ldots, \beta_{n}^{*}\right)$ and (i) $\boldsymbol{\beta}, \boldsymbol{\beta}^{*} \in \mathbb{D}_{+}$and $\boldsymbol{\alpha} \in \mathbb{E}_{+}$, then $X_{n: n} \leq_{\mathrm{rh}} X_{n: n}^{*}$; (ii) $\boldsymbol{\beta}, \boldsymbol{\beta}^{*}, \boldsymbol{\alpha} \in \mathbb{D}_{+}$, then $X_{n: n} \geq_{\mathrm{rh}} X_{n: n}^{*}$

Proof. Assuming $g\left(x_{i}\right)=x_{i}, u_{i}=\frac{\alpha_{i} x^{\gamma} e^{\lambda x}}{e^{\alpha_{i} x^{\gamma} e^{\lambda x}}-1}$ and

$$
\varphi(\boldsymbol{\beta})=\frac{(\lambda x+\gamma)}{x} \sum_{i=1}^{n} u_{i} g\left(\beta_{i}\right)
$$

and by noting the fact that $u_{i} \in \mathbb{D}_{+}\left(u_{i} \in \mathbb{E}_{+}\right)$whenever $\alpha_{i} \in \mathbb{E}_{+}\left(\alpha_{i} \in \mathbb{D}_{+}\right)$ (by Lemma 8 of Balakrishnan et al. (2018)) and using Theorem 3.1 and Theorem 3.2 of Kundu et al. (2016) it can be proved that $\tilde{r}_{X_{n: n}}(x)$ is Schur convex under condition (i), and Schur concave under condition (ii). This proves the result.

The following theorem follows from Lemma 2 and Theorem 5.

Theorem 6. For $i=1, \ldots, n$, let $X_{i}$ and $X_{i}^{*}$ be two sets of mutually independent random variables with $X_{i} \sim \operatorname{GMW}\left(\alpha_{i}, \gamma, \lambda, \beta_{i}\right)$ and $X_{i}^{*} \sim \operatorname{GMW}\left(\alpha_{i}, \gamma, \lambda, \beta_{i}^{*}\right)$.

(i) If $\boldsymbol{\beta}, \boldsymbol{\beta}^{*} \in \mathbb{D}_{+}$and $\boldsymbol{\alpha} \in \mathbb{E}_{+}$, then $\left(\beta_{1}, \ldots, \beta_{n}\right) \preceq_{w}\left(\beta_{1}^{*}, \ldots, \beta_{n}^{*}\right)$ implies $X_{n: n} \leq_{\mathrm{rh}} X_{n: n}^{*} ;$ (ii) If $\boldsymbol{\beta}, \boldsymbol{\beta}^{*}, \boldsymbol{\alpha} \in \mathbb{D}_{+}$, then $\left(\beta_{1}, \ldots, \beta_{n}\right) \stackrel{\mathrm{w}}{\preceq}\left(\beta_{1}^{*}, \ldots, \beta_{n}^{*}\right)$ implies $X_{n: n} \geq_{\mathrm{rh}} X_{n: n}^{*}$

The following result considers the comparison of the lifetimes of two series systems in terms of the usual stochastic order when the shape parameters $(\alpha)$ of the distribution of component lifetimes of a system is weakly majorized by the shape parameters $\left(\alpha^{*}\right)$ of the other system.

Theorem 7. Let $X_{1}, \ldots, X_{n}$ be a set of independent random variables with $X_{i} \sim \operatorname{GMW}\left(\alpha_{i}, \gamma, \lambda, \beta\right), i=1, \ldots, n$. Further, let $X_{1}^{*}, \ldots, X_{n}^{*}$ be another set 
of independent random variables with $X_{i}^{*} \sim \operatorname{GMW}\left(\alpha_{i}^{*}, \gamma, \lambda, \beta\right), i=1, \ldots, n$. Then, (i) for $\left(\alpha_{1}, \ldots, \alpha_{n}\right) \preceq_{w}\left(\alpha_{1}^{*}, \ldots, \alpha_{n}^{*}\right)$, we have $X_{1: n} \geq_{\text {st }} X_{1: n}^{*}$ if $\beta \geq 1$; (ii) $\left(\alpha_{1}, \ldots, \alpha_{n}\right) \stackrel{\mathrm{w}}{\preceq}\left(\alpha_{1}^{*}, \ldots, \alpha_{n}^{*}\right)$, we have $X_{1: n} \leq_{s t} X_{1: n}^{*}$ if $0<\beta \leq 1$.

Proof. To prove the first part (second part), it is sufficient to show that, for $\beta \geq 1(0<\beta \leq 1)$, the survival function of $X_{1: n}$ is Schur-concave (Schurconvex $)$ in $\left(\alpha_{1}, \ldots, \alpha_{n}\right)$ and is decreasing in $\alpha_{i}, i=1, \ldots, n$.. The survival function of $X_{1: n}$ is given by

$$
\bar{F}_{X_{1: n}}(x)=\prod_{i=1}^{n}\left(1-\left(1-\exp \left(-\alpha_{i} x^{\gamma} \exp (\lambda x)\right)\right)^{\beta}\right), x>0 .
$$

Differentiating (2) partially with respect to $\alpha_{i}, i=1, \ldots, n$, we get

$$
\frac{\partial \bar{F}_{X_{1: n}}(x)}{\partial \alpha_{i}}=-x^{\gamma} \exp (\lambda x) \bar{F}_{X_{1: n}}(x) \frac{\beta \exp \left(-\alpha_{i} x^{\gamma} \exp (\lambda x)\right)}{1-\left(1-\exp \left(-\alpha_{i} x^{\gamma} \exp (\lambda x)\right)\right)} \leq 0 .
$$

Thus, the survival function of $X_{1: n}$ given by (2) is decreasing in $\alpha_{i}, i=$ $1, \ldots, n$. To prove its Schur-concavity (Schur-convexity) see Theorem 2 of Balakrishnan et al. (2018).

Note that $\left(\alpha_{1}, \ldots, \alpha_{n}\right) \stackrel{\mathrm{m}}{\preceq}\left(\alpha_{1}^{*}, \ldots, \alpha_{n}^{*}\right)$ implies both $\left(\alpha_{1}, \ldots, \alpha_{n}\right) \preceq_{\mathrm{w}}\left(\alpha_{1}^{*}, \ldots, \alpha_{n}^{*}\right)$ and $\left(\alpha_{1}, \ldots, \alpha_{n}\right) \stackrel{\mathrm{w}}{\preceq}\left(\alpha_{1}^{*}, \ldots, \alpha_{n}^{*}\right)$, Theorem 7 substantially improves Theorem 2 of Balakrishnan et al. (2018).

Example 2. (i) Let $\left(X_{1}, X_{2}, X_{3}\right)\left(\left(X_{1}^{*}, X_{2}^{*}, X_{3}^{*}\right)\right)$ be a vector of independent heterogeneous GMW random variables. Set $\gamma=1.3, \lambda=1.6, \beta=1.3$, $\left(\alpha_{1}, \alpha_{2}, \alpha_{3}\right)=(5,2,0.2)$ and $\left(\alpha_{1}^{*}, \alpha_{2}^{*}, \alpha_{3}^{*}\right)=(8,3,0.3)$. Obviously $\left(\alpha_{1}, \alpha_{2}, \alpha_{3}\right) \preceq_{w}$ $\left(\alpha_{1}^{*}, \alpha_{2}^{*}, \alpha_{3}^{*}\right)$, and $X_{1: 3} \geq_{s t} X_{1: 3}^{*}$, as can be seen in Fig. $2(\mathrm{~A})$. (ii)Let $\left(X_{1}, X_{2}, X_{3}\right)$ $\left(\left(X_{1}^{*}, X_{2}^{*}, X_{3}^{*}\right)\right)$ be a vector of independent heterogeneous GMW random variables. Set $\gamma=1.3, \lambda=1.6, \beta=0.3,\left(\alpha_{1}, \alpha_{2}, \alpha_{3}\right)=(0.2,4,9)$ and $\left(\alpha_{1}^{*}, \alpha_{2}^{*}, \alpha_{3}^{*}\right)=(0.1,1,6)$. Obviously $\left(\alpha_{1}, \alpha_{2}, \alpha_{3}\right) \stackrel{\mathrm{w}}{\preceq}\left(\alpha_{1}^{*}, \alpha_{2}^{*}, \alpha_{3}^{*}\right)$, and $X_{1: 3} \leq_{s t}$ $X_{1: 3}^{*}$, as can be seen in Fig. $2(\mathrm{~B})$.

Naturally, one may wonder whether the following statement is actually also true: For $0<\beta \leq 1,\left(\alpha_{1}, \ldots, \alpha_{n}\right) \stackrel{\mathrm{p}}{\preceq}\left(\alpha_{1}^{*}, \ldots, \alpha_{n}^{*}\right)$ gives rise to the usual stochastic order between $X_{1: n}$ and $X_{1: n}^{*}$. The following example gives negative answer to these two conjecture. 


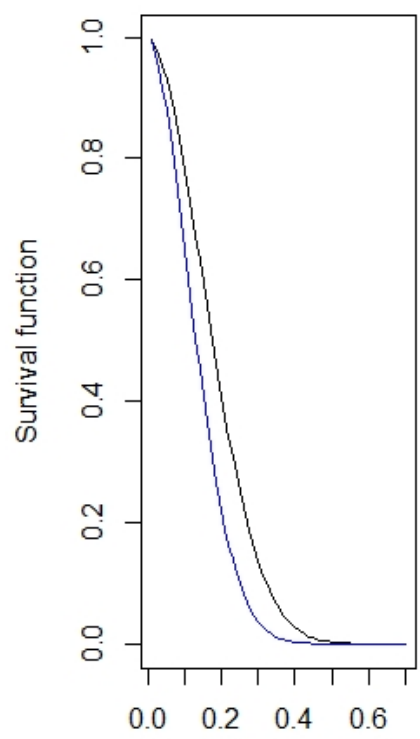

(A)

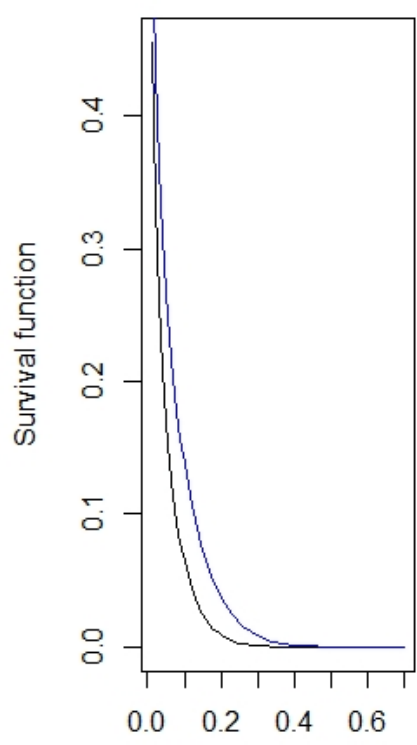

(B)

Figure 2. Plot of the survival functions of $X_{1: 3}$ (black line) and $X_{1: 3}^{*}$ (blue line)

Example 3. Let $\left(X_{1}, X_{2}\right)\left(\left(X_{1}^{*}, X_{2}^{*}\right)\right)$ be a vector of independent heterogeneous GMW random variables. Set $\beta=0.5, \gamma=2$ and $\lambda=3$. For $\left(\alpha_{1}, \alpha_{2}\right)=(2,3) \stackrel{\mathrm{p}}{\preceq}(1,5.5)=\left(\alpha_{1}^{*}, \alpha_{2}^{*}\right), X_{1: 2} \geq_{\text {st }} X_{1: 2}^{*}$, as can be seen in Fig. 3 (A); however, for $\left(\alpha_{1}, \alpha_{2}\right)=(1.1,6) \stackrel{\mathrm{p}}{\preceq}(1,2.25)=\left(\alpha_{1}^{*}, \alpha_{2}^{*}\right), X_{1: 2} \leq_{\mathrm{st}} X_{1: 2}^{*}$, as can be seen in Fig. 3 (B) So, $\left(\alpha_{1}, \alpha_{2}\right) \stackrel{\mathrm{p}}{\preceq}\left(\alpha_{1}^{*}, \alpha_{2}^{*}\right)$ implies neither $X_{1: 2} \leq_{\text {st }} X_{1: 2}^{*}$ nor $X_{1: 2} \geq_{\text {st }} X_{1: 2}^{*}$ for $0<\beta \leq 1$.

Now, we discuss stochastic comparison between the lifetimes of two series systems in the sense of the hazard rate order.

Theorem 8. Let $X_{1}, \ldots, X_{n}$ be a set of independent random variables with $X_{i} \sim \operatorname{GMW}\left(\alpha, \gamma, \lambda, \beta_{i}\right), i=1, \ldots, n$. Further, let $X_{1}^{*}, \ldots, X_{n}^{*}$ be another set of independent random variables with $X_{i}^{*} \sim \operatorname{GMW}\left(\alpha, \gamma, \lambda, \beta_{i}^{*}\right), i=1, \ldots, n$. If $\left.\left(\beta_{1}, \ldots, \beta_{n}\right) \stackrel{\mathrm{w}}{\succeq} \beta_{1}^{*}, \ldots, \beta_{n}^{*}\right)$, then $X_{1: n} \leq_{\mathrm{hr}} X_{1: n}^{*}$. 


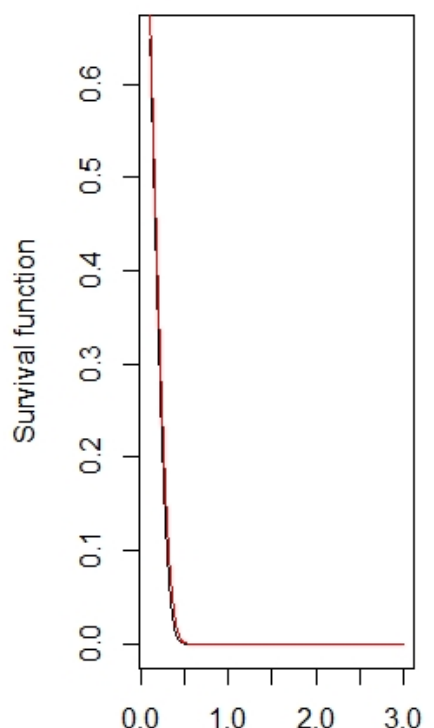

(A)

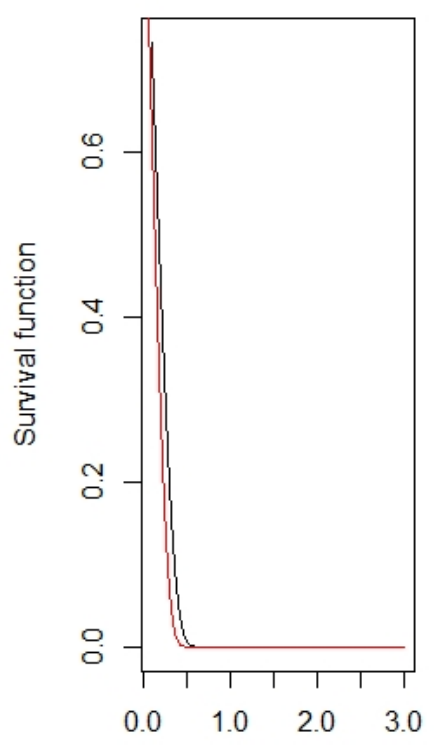

(B)

Figure 3. Plot of the survival functions of $X_{1: 2}$ (red line) and $X_{1: 3}^{*}$ (black line)

Proof. The hazard rate of $X_{1: n}$ can be obtained as

$$
\begin{aligned}
h_{X_{1: n}}(x)= & \left(\alpha x^{\gamma} \exp (\lambda x)\right)\left(\lambda+\frac{\gamma}{x}\right)\left(\exp \left(-\alpha x^{\gamma} \exp (\lambda x)\right)\right) \times \\
& \sum_{i=1}^{n} g\left(\beta_{i}, 1-\exp \left(-\alpha x^{\gamma} \exp (\lambda x)\right)\right) .
\end{aligned}
$$

where $g$ is given as in Lemma 3. Using Lemma 2, it is enough to show that the function $h_{X_{1: n}}(x)$ is Schur-convex and decreasing in $\beta_{i}$ 's. The partial derivatives of $h_{X_{1: n}}(x)$ with respect to $\beta_{i}$ are

$$
\frac{\partial h_{X_{1: n}}(x)}{\partial \beta_{i}}=
$$




$$
\left(\alpha x^{\gamma} \exp (\lambda x)\right)\left(\lambda+\frac{\gamma}{x}\right)\left(\exp \left(-\alpha x^{\gamma} \exp (\lambda x)\right)\right) \frac{\partial g\left(\beta_{i}, 1-\exp \left(-\alpha x^{\gamma} \exp (\lambda x)\right)\right)}{\partial \beta_{i}} .
$$

Furthermore, from Lemma $3, g(\beta, x)$ is a decreasing function of $\beta$. Using this, we obtain $\frac{\partial h_{X_{1: n}}(x)}{\partial \beta_{i}} \leq 0$. Thus, the hazard rate of $X_{1: n}$ is decreasing in $\beta_{i}, i=1, \ldots, n$. To prove its Schur-convexity see Theorem 3 of Balakrishnan et al. (2018).

The following result considers the comparison of the lifetimes of parallel systems in terms of the usual stochastic order with respect to the scale parameter $\lambda$.

Theorem 9. Let $X_{1}, \ldots, X_{n}$ be a set of independent random variables with $X_{i} \sim \operatorname{GMW}\left(\alpha, \gamma, \lambda_{i}, \beta\right), i=1, \ldots, n$. Further, let $X_{1}^{*}, \ldots, X_{n}^{*}$ be another set of independent random variables with $X_{i}^{*} \sim \operatorname{GMW}\left(\alpha, \gamma, \lambda_{i}^{*}, \beta\right), i=1, \ldots, n$. Then, for $\left(\lambda_{1}, \ldots, \lambda_{n}\right) \stackrel{\mathrm{w}}{\preceq}\left(\lambda_{1}^{*}, \ldots, \lambda_{n}^{*}\right)$, we have $X_{n: n} \leq_{\text {st }} X_{n: n}^{*}$.

Proof. The cdf of $X_{n: n}$ is given by

$$
F_{X_{n: n}}(x)=\prod_{i=1}^{n}\left(1-\exp \left(-\alpha x^{\gamma} \exp \left(\lambda_{i} x\right)\right)^{\beta}\right), x>0 .
$$

Using Lemma 2, it is enough to show that the function $F_{X_{n: n}}(x)$ is Schurconcave and increasing in $\lambda_{i}$ 's. Differentiating (5) partially with respect to $\lambda_{i}, i=1, \ldots, n$, we get

$$
\frac{\partial F_{X_{n: n}}(x)}{\partial \lambda_{i}}=x \beta F_{X_{n: n}}(x) \frac{\alpha x^{\gamma} \exp \left(\lambda_{i} x\right)}{\exp \left(\alpha x^{\gamma} \exp \left(\lambda_{i} x\right)\right)-1} \geq 0 .
$$

Thus, the cdf of $X_{n: n}$ is increasing in $\lambda_{i}, i=1, \ldots, n$. To prove its Schurconcavity see Theorem 4 of Balakrishnan et al. (2018).

The next result considers the comparison of the lifetimes of series systems in terms of the usual stochastic order with respect to the scale parameter $\lambda$.

Theorem 10. Let $X_{1}, \ldots, X_{n}$ be a set of independent random variables with $X_{i} \sim \operatorname{GMW}\left(\alpha, \gamma, \lambda_{i}, \beta\right), i=1, \ldots, n$. Further, let $X_{1}^{*}, \ldots, X_{n}^{*}$ be another set of independent random variables with $X_{i}^{*} \sim \operatorname{GMW}\left(\alpha, \gamma, \lambda_{i}^{*}, \beta\right), i=1, \ldots, n$. Then, for $\left(\lambda_{1}, \ldots, \lambda_{n}\right) \preceq_{\mathrm{w}}\left(\lambda_{1}^{*}, \ldots, \lambda_{n}^{*}\right)$, we have $X_{1: n} \geq_{\mathrm{st}} X_{1: n}^{*}$ if $\beta \geq 1$. 
Proof. The survival function of $X_{1: n}$ is given by

$$
\bar{F}_{X_{1: n}}(x)=\prod_{i=1}^{n}\left(1-\left(1-\exp \left(-\alpha x^{\gamma} \exp \left(\lambda_{i} x\right)\right)\right)^{\beta}\right), x>0 .
$$

Its easy to see that $\bar{F}_{X_{1: n}}(x)$ is decreasing in $\lambda_{i}, i=1, \ldots, n$. To prove its Schur-concavity see Theorem 4 of Balakrishnan et al. (2018).

\subsection{Interdependent Variables with Archimedean Copulas}

Recently, some authors paid their attention to comparing order statistics of dependent samples. For instance, see, Bashkar et al. (2017), Li and $\mathrm{Li}$ (2015), Li and Fang (2015) and Fang et al. (2015). In this section, we derive some new results on the usual stochastic order between the minimum of order statistics of two heterogeneous random vectors with the dependent components having Archimedean copula structure with GMW marginals. Before going into the details, let us recall one important general family of distributions. We say that random variable $X$ belongs to the Exponentiated Scale (ES) family of distributions if $X \sim H(x)=[G(\lambda x)]^{\alpha}$, where $\alpha, \lambda>0$ and $G$ is called the baseline distribution function which we assume that is absolutely continuous. In the sequel, we denote this family by $\operatorname{ES}(\alpha, \lambda)$. Bashkar et al. (2017) proved the following general result.

Theorem 11. Suppose for $i=1, \ldots, n, X_{i} \sim \operatorname{ES}\left(\alpha_{i}, \lambda\right)$ and $X_{i}^{*} \sim \operatorname{ES}\left(\alpha_{i}^{*}, \lambda\right)$ share a common Archimedean survival copula with generator $\phi$. Then, $X_{1: n} \leq_{\text {st }} X_{1: n}^{*}$ if $\left(\alpha_{1}, \ldots, \alpha_{n}\right) \stackrel{\mathrm{w}}{\succeq}\left(\alpha_{1}^{*}, \ldots, \alpha_{n}^{*}\right)$.

The following result, for the GMW samples with Archimedean survival copulas, follows immediately from Theorem 11 .

Theorem 12. Suppose for $i=1, \ldots, n, X_{i} \sim \operatorname{GMW}\left(\alpha, \gamma, \lambda, \beta_{i}\right)$ and $X_{i}^{*} \sim$ $\operatorname{GMW}\left(\alpha, \gamma, \lambda, \beta_{i}^{*}\right)$ share a common Archimedean survival copula with generator $\phi$. Then, $X_{1: n} \leq_{\text {st }} X_{1: n}^{*}$ if $\left(\beta_{1}, \ldots, \beta_{n}\right) \stackrel{\mathrm{w}}{\succeq}\left(\beta_{1}^{*}, \ldots, \beta_{n}^{*}\right)$.

In the sequel, by $\boldsymbol{X} \sim \operatorname{GMW}(\boldsymbol{\beta}, \alpha, \gamma, \lambda, \phi)$ we mean that $\mathbf{X}$ has the Archimedean copula with generator $\phi$ and for $i=1, \ldots, n, X_{i} \sim G M W\left(\beta_{i}, \alpha, \gamma, \lambda\right)$. The smallest order statistic $X_{1: n}$ of the sample $\boldsymbol{X} \sim \operatorname{GMW}(\boldsymbol{\beta}, \alpha, \gamma, \lambda, \phi)$ has the survival function

$$
\bar{G}_{X_{1: n}}(x)=\phi\left(\sum_{i=1}^{n} \psi\left(1-(F(\alpha, \gamma, \lambda, x))^{\beta_{i}}\right)\right) \equiv J(\boldsymbol{\beta}, \alpha, \gamma, \lambda, x, \phi)
$$


where, $F(\alpha, \gamma, \lambda, x)=1-\exp \left(-\alpha x^{\gamma} \exp (\lambda x)\right)$.

Theorem 13. For $\boldsymbol{X} \sim \operatorname{GMW}\left(\boldsymbol{\beta}, \alpha, \gamma, \lambda, \phi_{1}\right)$ and $\boldsymbol{X}^{*} \sim \operatorname{GMW}\left(\boldsymbol{\beta}^{*}, \alpha, \gamma, \lambda, \phi_{2}\right)$, if $\psi_{2} \circ \phi_{1}$ is super-additive, then $\left(\beta_{1}, \ldots, \beta_{n}\right) \stackrel{\mathrm{w}}{\succeq}\left(\beta_{1}^{*}, \ldots, \beta_{n}^{*}\right)$ implies $X_{1: n} \leq_{\mathrm{st}}$ $X_{1: n}^{*}$.

Proof. According to Equation (8), $X_{1: n}$ and $X_{1: n}^{*}$ have their respective survival functions $J\left(\boldsymbol{\beta}, \alpha, \gamma, \lambda, x, \phi_{1}\right)$ and $J\left(\boldsymbol{\beta}, \alpha, \gamma, \lambda, x, \phi_{2}\right)$ for $x \geq 0$. First we show that $J\left(\boldsymbol{\beta}, \alpha, \gamma, \lambda, x, \phi_{1}\right)$ is increasing and Schur-concave function of $\beta_{i}, i=1, \ldots, n$. Since $\phi_{1}$ is decreasing, we have

$$
\begin{gathered}
\frac{\partial J\left(\boldsymbol{\beta}, \alpha, \gamma, \lambda, x, \phi_{1}\right)}{\partial \alpha_{i}}=- \\
\frac{(F(\alpha, \gamma, \lambda, x))^{\beta_{i}} \log (F(\alpha, \gamma, \lambda, x)) \phi_{1}^{\prime}\left(\sum_{i=1}^{n} \psi\left(1-(F(\alpha, \gamma, \lambda, x))^{\beta_{i}}\right)\right)}{\phi_{1}^{\prime}\left(\psi\left(1-(F(\alpha, \gamma, \lambda, x))^{\beta_{i}}\right)\right)} \geq 0, \\
\text { for all } x>0,
\end{gathered}
$$

That is, $J\left(\boldsymbol{\beta}, \alpha, \gamma, \lambda, x, \phi_{1}\right)$ is increasing in $\beta_{i}$ for $i=1, \ldots, n$.

To prove its Schur-concavety, from lemma 1, we need to show that for $i \neq j$,

$$
\left(\beta_{i}-\beta_{j}\right)\left(\frac{\partial J\left(\boldsymbol{\beta}, \alpha, \gamma, \lambda, x, \phi_{1}\right)}{\partial \beta_{i}}-\frac{\partial J\left(\boldsymbol{\beta}, \alpha, \gamma, \lambda, x, \phi_{1}\right)}{\partial \beta_{j}}\right) \leq 0,
$$

that is, for $i \neq j$,

$$
\begin{gathered}
\left.-\log (F(\alpha, \gamma, \lambda, x)) \phi_{1}^{\prime}\left(\sum_{i=1}^{n} \psi_{1}\left(1-(F(\alpha, \gamma, \lambda, x))^{\beta_{i}}\right)\right)\right)\left(\beta_{i}-\beta_{j}\right) \times \\
\left(\frac{(F(\alpha, \gamma, \lambda, x))^{\beta_{i}}}{\phi_{1}^{\prime}\left(\psi_{1}\left(1-(F(\alpha, \gamma, \lambda, x))^{\beta_{i}}\right)\right)}-\frac{(F(\alpha, \gamma, \lambda, x))^{\beta_{j}}}{\phi_{1}^{\prime}\left(\psi_{1}\left(1-(F(\alpha, \gamma, \lambda, x))^{\beta_{j}}\right)\right)}\right) \leq 0 .
\end{gathered}
$$

Now, let us consider the function $g(\beta)=\frac{(F(\alpha, \gamma, \lambda, x))^{\beta_{i}}}{\phi_{1}^{\prime}\left(\psi\left(1-(F(\alpha, \gamma, \lambda, x))^{\beta_{i}}\right)\right)} \cdot g(\beta)$ is increasing with respect to $\beta$, from which it follows that (9) holds. According to Lemma $2, \boldsymbol{\beta} \stackrel{\mathrm{w}}{\succeq} \boldsymbol{\beta}^{*}$ implies $J\left(\boldsymbol{\beta}, \alpha, \gamma, \lambda, x, \phi_{1}\right) \leq J\left(\boldsymbol{\beta}^{*}, \alpha, \gamma, \lambda, x, \phi_{1}\right)$. On the other hand, since $\psi_{2} \circ \phi_{1}$ is super-additive by Lemma A.1. of Li and Fang 
(2015), we have $J\left(\boldsymbol{\beta}^{*}, \alpha, \gamma, \lambda, x, \phi_{1}\right) \leq J\left(\boldsymbol{\beta}^{*}, \alpha, \gamma, \lambda, x, \phi_{2}\right)$. So, it holds that

$$
J\left(\boldsymbol{\beta}, \alpha, \gamma, \lambda, x, \phi_{1}\right) \leq J\left(\boldsymbol{\beta}^{*}, \alpha, \gamma, \lambda, x, \phi_{1}\right) \leq J\left(\boldsymbol{\beta}^{*}, \alpha, \gamma, \lambda, x, \phi_{2}\right) .
$$

That is, $X_{1: n} \leq_{\text {st }} X_{1: n}^{*}$.

Theorem 13 generalizes the result of the Theorem 12 to GMW samples with not necessarily a common dependence structure. Theorem 14 generalizes the result of Bashkar et al. (2017) to ES samples with not necessarily a common dependence structure. The smallest order statistic $X_{1: n}$ of the sample $\boldsymbol{X} \sim \operatorname{ES}(\boldsymbol{\alpha}, \lambda, \phi)$ gets survival function

$$
\bar{G}_{X_{1: n}}(x)=\phi\left(\sum_{i=1}^{n} \psi\left(1-G^{\alpha_{i}}(\lambda x)\right)\right)=J_{1}(\boldsymbol{\alpha}, \lambda, x, \phi)
$$

Theorem 14. For $\boldsymbol{X} \sim \operatorname{ES}\left(\boldsymbol{\alpha}, \lambda, \phi_{1}\right)$ and $\boldsymbol{X}^{*} \sim \operatorname{ES}\left(\boldsymbol{\alpha}^{*}, \lambda, \phi_{2}\right)$, if $\psi_{2} \circ \phi_{1}$ is super-additive, then $\boldsymbol{\alpha} \stackrel{\mathrm{w}}{\succeq} \boldsymbol{\alpha}^{*}$ implies $X_{1: n} \leq_{\mathrm{st}} X_{1: n}^{*}$.

Proof. According to Equation (10), $X_{1: n}$ and $X_{1: n}^{*}$ have their respective survival functions $J_{1}\left(\boldsymbol{\alpha}, \lambda, x, \phi_{1}\right)$ and $J_{1}\left(\boldsymbol{\alpha}^{*}, \lambda, x, \phi_{2}\right)$ for $x \geq 0$.

First we show that $J_{1}\left(\boldsymbol{\alpha}, \lambda, x, \phi_{1}\right)$ is increasing and Schur-concave function of $\alpha_{i}, i=1, \ldots, n$. Since $\phi_{1}$ is decreasing, we have

$$
\begin{gathered}
\frac{\partial J_{1}\left(\boldsymbol{\alpha}, \lambda, x, \phi_{1}\right)}{\partial \alpha_{i}}=-\frac{G^{\alpha_{i}}(\lambda x) \log (G(\lambda x)) \phi_{1}^{\prime}\left(\sum_{i=1}^{n} \psi\left(1-G^{\alpha_{i}}(\lambda x)\right)\right)}{\phi_{1}^{\prime}\left(\psi\left(1-G^{\alpha_{i}}(\lambda x)\right)\right)} \geq 0, \\
\text { for all } \quad x>0,
\end{gathered}
$$

That is, $J_{1}\left(\boldsymbol{\alpha}, \lambda, x, \phi_{1}\right)$ is increasing in $\alpha_{i}$ for $i=1, \ldots, n$.

To prove its Schur-concavety, it follows from lemma 1 that we have to show that for $i \neq j$,

$$
\left(\alpha_{i}-\alpha_{j}\right)\left(\frac{\partial J_{1}\left(\boldsymbol{\alpha}, \lambda, x, \phi_{1}\right)}{\partial \alpha_{i}}-\frac{\partial J_{1}\left(\boldsymbol{\alpha}, \lambda, x, \phi_{1}\right)}{\partial \alpha_{j}}\right) \leq 0
$$

that is, for $i \neq j$,

$$
\left.-\log (G(\lambda x)) \phi_{1}^{\prime}\left(\sum_{i=1}^{n} \psi_{1}\left(1-G^{\alpha_{i}}(\lambda x)\right)\right)\right)\left(\alpha_{i}-\alpha_{j}\right)
$$




$$
\left(\frac{G^{\alpha_{i}}(\lambda x)}{\phi_{1}^{\prime}\left(\psi_{1}\left(1-G^{\alpha_{i}}(\lambda x)\right)\right)}-\frac{G^{\alpha_{j}}(\lambda x)}{\phi_{1}^{\prime}\left(\psi_{1}\left(1-G^{\alpha_{j}}(\lambda x)\right)\right)}\right) \leq 0 .
$$

Now, let us consider the function $g(\alpha)=\frac{G^{\alpha}(\lambda x)}{\phi^{\prime}\left(\psi\left(1-G^{\alpha}(\lambda x)\right)\right)}$. Taking derivative with respect to $\alpha$, we get

$$
\begin{aligned}
& g^{\prime}(\alpha) \stackrel{\text { sgn }}{=} G^{\alpha}(\lambda x) \log (G(\lambda x)) \phi^{\prime}\left(\psi\left(1-G^{\alpha}(\lambda x)\right)\right) \\
& +\frac{G^{2 \alpha}(\lambda x) \log (G F(\lambda x))}{\phi^{\prime}\left(\psi\left(1-G^{\alpha}(\lambda x)\right)\right)} \phi^{\prime \prime}\left(\psi\left(1-G^{\alpha}(\lambda x)\right)\right) \geq 0 .
\end{aligned}
$$

Thus, $g(\alpha)$ is increasing with respect to $\alpha$, from which it follows that (11) holds. According to Lemma $2 \boldsymbol{\alpha} \succeq \boldsymbol{\alpha}^{*}$ implies $J_{1}\left(\boldsymbol{\alpha}, \lambda, x, \phi_{1}\right) \leq J_{1}\left(\boldsymbol{\alpha}^{*}, \lambda, x, \phi_{1}\right)$. On the other hand, since $\psi_{2} \circ \phi_{1}$ is super-additive by Lemma A.1. of Li and Fang (2015), we have $J_{1}\left(\boldsymbol{\alpha}^{*}, \lambda, x, \phi_{1}\right) \leq J_{1}\left(\boldsymbol{\alpha}^{*}, \lambda, x, \phi_{2}\right)$. So, it holds that

$$
J_{1}\left(\boldsymbol{\alpha}, \lambda, x, \phi_{1}\right) \leq J_{1}\left(\boldsymbol{\alpha}^{*}, \lambda, x, \phi_{1}\right) \leq J_{1}\left(\boldsymbol{\alpha}^{*}, \lambda, x, \phi_{2}\right) .
$$

That is, $X_{1: n} \leq_{\mathrm{st}} X_{1: n}^{*}$.

Note that if in Theorem 14, we take $\lambda=1$, then we get the following result for the proportional reversed hazards $(\mathrm{PRH})$ model.

Corollary 1. Suppose $\boldsymbol{X} \sim P R H\left(\boldsymbol{\alpha}, \phi_{1}\right)$ and $\boldsymbol{X}^{*} \sim P R H\left(\boldsymbol{\alpha}^{*}, \phi_{2}\right)$ and $\phi_{2} \circ \psi_{1}$ is super-additive. Then $\boldsymbol{\alpha} \succeq \boldsymbol{\alpha}^{*}$ implies $X_{1: n} \leq_{\mathrm{st}} X_{1: n}^{*}$.

In the ES family, Bashkar et al. (2018) obtained some sufficient conditions for the comparison of parallel systems under some well-known stochastic orderings. Their results are stated in the following theorem.

Theorem 15. For $\boldsymbol{X} \sim \operatorname{ES}\left(\boldsymbol{\alpha}, \lambda, \phi_{1}\right)$ and $\boldsymbol{X}^{*} \sim \operatorname{ES}\left(\boldsymbol{\alpha}^{*}, \lambda, \phi_{2}\right)$,

(i) if $\phi_{1}$ or $\phi_{2}$ is log-convex, and $\psi_{2} \circ \phi_{1}$ is super-additive, then $\left(\alpha_{1}, \ldots, \alpha_{n}\right) \succeq_{\mathrm{w}}$ $\left(\alpha_{1}^{*}, \ldots, \alpha_{n}^{*}\right)$ implies $X_{n: n} \geq_{\text {st }} X_{n: n}^{*}$;

(ii) if $\phi_{1}$ or $\phi_{2}$ is log-concave, and $\psi_{1} \circ \phi_{2}$ is super-additive, then $\left(\alpha_{1}, \ldots, \alpha_{n}\right) \stackrel{\mathrm{w}}{\succeq}$ $\left(\alpha_{1}^{*}, \ldots, \alpha_{n}^{*}\right)$ implies $X_{n: n} \leq_{\mathrm{st}} X_{n: n}^{*}$.

The following result follows immediately from Theorem 15 .

Theorem 16. For $\boldsymbol{X} \sim \operatorname{GMW}\left(\boldsymbol{\beta}, \alpha, \gamma, \lambda, \phi_{1}\right)$ and $\boldsymbol{X}^{*} \sim \operatorname{GMW}\left(\boldsymbol{\beta}^{*}, \alpha, \gamma, \lambda, \phi_{2}\right)$, 
(i) if $\phi_{1}$ or $\phi_{2}$ is log-convex, and $\psi_{2} \circ \phi_{1}$ is super-additive, then $\left(\beta_{1}, \ldots, \beta_{n}\right) \succeq_{\mathrm{w}}$ $\left(\beta_{1}^{*}, \ldots, \beta_{n}^{*}\right)$ implies $X_{n: n} \geq_{\mathrm{st}} X_{n: n}^{*}$;

(ii) if $\phi_{1}$ or $\phi_{2}$ is log-concave, and $\psi_{1} \circ \phi_{2}$ is super-additive, then $\left(\beta_{1}, \ldots, \beta_{n}\right) \stackrel{\mathrm{w}}{\succeq}$ $\left(\beta_{1}^{*}, \ldots, \beta_{n}^{*}\right)$ implies $X_{n: n} \leq_{\mathrm{st}} X_{n: n}^{*}$.

The following theorem provides some sufficient conditions under which weakly supermajorized order between the scale parameter vectors implies the usual stochastic order between the largest order statistics.

Theorem 17. For $\boldsymbol{X} \sim \operatorname{GMW}\left(\beta, \boldsymbol{\alpha}, \gamma, \lambda, \phi_{1}\right)$ and $\boldsymbol{X}^{*} \sim \operatorname{GMW}\left(\beta, \boldsymbol{\alpha}^{*}, \gamma, \lambda, \phi_{2}\right)$, if $\phi_{1}$ or $\phi_{2}$ is log-convex, and $\psi_{2} \circ \phi_{1}$ is super-additive, then $\left(\alpha_{1}, \ldots, \alpha_{n}\right) \stackrel{\mathrm{W}}{\succeq}$ $\left(\alpha_{1}^{*}, \ldots, \alpha_{n}^{*}\right)$ implies $X_{n: n} \geq_{\text {st }} X_{n: n}^{*}$.

Proof. The largest order statistic $X_{n: n}$ of the sample $\boldsymbol{X} \sim \operatorname{GMW}\left(\beta, \boldsymbol{\alpha}, \gamma, \lambda, \phi_{1}\right)$ gets distribution function

$$
F_{X_{n: n}}(x)=\phi_{1}\left(\sum_{i=1}^{n} \psi_{1}\left(\left(1-\exp \left(-\alpha_{i} x^{\gamma} \exp (\lambda x)\right)\right)^{\beta}\right)\right)=J_{2}\left(\beta, \boldsymbol{\alpha}, \gamma, \lambda, x, \phi_{1}\right)
$$

We only prove the case that $\phi_{1}$ is log-convex, and the other case can be finished similarly. First we show that $J_{2}\left(\beta, \boldsymbol{\alpha}, \gamma, \lambda, x, \phi_{1}\right)$ is increasing and Schur-concave function of $\alpha_{i}, i=1, \ldots, n$. Since $\phi_{1}$ is decreasing, we have

$$
\begin{gathered}
\frac{\partial J_{2}\left(\beta, \boldsymbol{\alpha}, \gamma, \lambda, x, \phi_{1}\right)}{\partial \alpha_{i}}=\beta x^{\gamma} e^{\lambda x} e^{-\alpha_{i} x^{\gamma} \exp (\lambda x)}\left(1-\exp \left(-\alpha_{i} x^{\gamma} \exp (\lambda x)\right)\right)^{\beta-1} \\
\times \frac{\phi_{1}^{\prime}\left(\sum_{i=1}^{n} \psi_{1}\left(\left(1-\exp \left(-\alpha_{i} x^{\gamma} \exp (\lambda x)\right)\right)^{\beta}\right)\right)}{\phi_{1}^{\prime}\left(\psi_{1}\left(\left(1-\exp \left(-\alpha_{i} x^{\gamma} \exp (\lambda x)\right)\right)^{\beta}\right)\right)} \geq 0, \\
\text { for all } x>0,
\end{gathered}
$$

That is, $J_{2}\left(\beta, \boldsymbol{\alpha}, \gamma, \lambda, x, \phi_{1}\right)$ is increasing in $\alpha_{i}$ for $i=1, \ldots, n$. Furthermore, for $i \neq j$,

$$
\begin{gathered}
\frac{\partial J_{2}\left(\beta, \boldsymbol{\alpha}, \gamma, \lambda, x, \phi_{1}\right)}{\partial \alpha_{i}}-\frac{\partial J_{2}\left(\beta, \boldsymbol{\alpha}, \gamma, \lambda, x, \phi_{1}\right)}{\left.\partial \alpha_{j}\right)}= \\
\beta x^{\gamma} e^{\lambda x} \phi_{1}^{\prime}\left(\sum_{i=1}^{n} \psi_{1}\left(\left(1-\exp \left(-\alpha_{i} x^{\gamma} \exp (\lambda x)\right)\right)^{\beta}\right)\right) \\
\times\left(\frac{e^{-\alpha_{i} x^{\gamma} \exp (\lambda x)}}{\left(1-e^{-\alpha_{i} x^{\gamma} \exp (\lambda x)}\right)} \times \frac{\phi_{1}\left(\psi_{1}\left(1-e^{-\alpha_{i} x^{\gamma} \exp (\lambda x)}\right)\right)}{\phi_{1}^{\prime}\left(\psi_{1}\left(1-e^{-\alpha_{i} x^{\gamma} \exp (\lambda x)}\right)\right)}-\right.
\end{gathered}
$$




$$
\frac{e^{-\alpha_{j} x^{\gamma} \exp (\lambda x)}}{\left(1-e^{-\alpha_{j} x^{\gamma} \exp (\lambda x)}\right)} \times \frac{\phi_{1}\left(\psi_{1}\left(1-e^{-\alpha_{j} x^{\gamma} \exp (\lambda x)}\right)\right)}{\phi_{1}^{\prime}\left(\psi_{1}\left(1-e^{-\alpha_{j} x^{\gamma} \exp (\lambda x)}\right)\right)} .
$$

Note that the log-convexity of $\phi_{1}$ implies the decreasing property of $\frac{\phi_{1}}{\phi_{1}^{\prime}}$. Since $\psi_{1}\left(1-e^{-\alpha x^{\gamma} \exp (\lambda x)}\right)$ is decreasing in $\alpha>0$, then $\frac{\phi_{1}\left(\psi_{1}\left(1-e^{-\alpha_{i} x^{\gamma} \exp (\lambda x)}\right)\right)}{\phi_{1}^{\prime}\left(\psi_{1}\left(1-e^{-\alpha_{i} x^{\gamma} \exp (\lambda x)}\right)\right)}$ is increasing in $\alpha>0$. Also $\frac{e^{-\alpha x^{\gamma} \exp (\lambda x)}}{\left(1-e^{-\alpha x^{\gamma} \exp (\lambda x)}\right)}$ is decreasing in $\alpha>0$, and thus $\frac{e^{-\alpha x^{\gamma} \exp (\lambda x)}}{\left(1-e^{-\alpha x^{\gamma} \exp (\lambda x)}\right)} \times \frac{\phi_{1}\left(\psi_{1}\left(1-e^{-\alpha x^{\gamma} \exp (\lambda x)}\right)\right)}{\phi_{1}^{\prime}\left(\psi_{1}\left(1-e^{-\alpha x^{\gamma} \exp (\lambda x)}\right)\right)}$ is increasing in $\alpha>0$. So, for $i \neq j$,

$$
\left(\alpha_{i}-\alpha_{j}\right)\left(\frac{\partial J_{2}\left(\beta, \boldsymbol{\alpha}, \gamma, \lambda, x, \phi_{1}\right)}{\partial \alpha_{i}}-\frac{\partial J_{2}\left(\beta, \boldsymbol{\alpha}, \gamma, \lambda, x, \phi_{1}\right)}{\left.\partial \alpha_{j}\right)}\right) \leq 0 .
$$

Then Schur-concavity of $J_{2}\left(\beta, \boldsymbol{\alpha}, \gamma, \lambda, x, \phi_{1}\right)$ follows from lemma 1. According to Lemma $2\left(\alpha_{1}, \ldots, \alpha_{n}\right) \stackrel{\mathrm{w}}{\succeq}\left(\alpha_{1}^{*}, \ldots, \alpha_{n}^{*}\right)$ implies $J_{2}\left(\beta, \boldsymbol{\alpha}, \gamma, \lambda, x, \phi_{1}\right) \leq$ $J_{2}\left(\beta, \boldsymbol{\alpha}^{*}, \gamma, \lambda, x, \phi_{1}\right)$. On the other hand, since $\psi_{2} \circ \phi_{1}$ is super-additive by Lemma A.1. of Li and Fang (2015), we have $J_{2}\left(\beta, \boldsymbol{\alpha}^{*}, \gamma, \lambda, x, \phi_{1}\right) \leq$ $J_{2}\left(\beta, \boldsymbol{\alpha}^{*}, \gamma, \lambda, x, \phi_{2}\right)$. So, it holds that

$$
J_{2}\left(\beta, \boldsymbol{\alpha}, \gamma, \lambda, x, \phi_{1}\right) \leq J_{2}\left(\beta, \boldsymbol{\alpha}^{*}, \gamma, \lambda, x, \phi_{1}\right) \leq J_{2}\left(\beta, \boldsymbol{\alpha}^{*}, \gamma, \lambda, x, \phi_{2}\right) .
$$

That is, $X_{n: n} \geq_{\text {st }} X_{n: n}^{*}$.

\section{Conclusions}

In this paper, we have considered series and parallel systems with independent heterogeneous generalized modified Weibull components. These comparisons are made with respect to usual stochastic, hazard rate and reversed hazard rate orderings. We also conducted stochastic comparison on the extreme order statistics from GMW samples with Archimedean survival copulas. The results of the paper extend some known results in the Balakrishnan et al. (2018).

\section{Acknowledgment}

The author would like to thank the referees for their valuable comments and suggestions, which resulted in an improvement in the presentation of this 
manuscript.

\section{References}

Balakrishnan, N., Nanda, P. and Kayal, S. (2018). Ordering of Series and Parallel Systems Comprising Heterogeneous Generalized Modified Weibull Components. Applied Stochastic Models in Business and Industry, 34, 816-834.

Carrasco, J.M., Ortega, E.M. and Cordeiro, G.M., (2008). A Generalized Modified Weibull Distribution for Lifetime Modeling. Computational Statistics and Data Analysis, 53, 450-462.

Balakrishnan, N., Haidari, A., and Masoumifard, K. (2014). Stochastic Comparisons of Series and Parallel Systems with Generalized Exponential Components. IEEE Transactions on Reliability, 64, 333-348.

Balakrishnan, N., and Zhao, P. (2013). Ordering Properties of Order Statistics from Heterogeneous Populations: a Review with an Emphasis on Some Recent Developments. Probability in the Engineering and Informational Sciences, 27, 403-443.

Bashkar, E., Torabi, H., and Roozegar, R. (2017). Stochastic Comparisons of Extreme Order Statistics in the Heterogeneous Exponentiated Scale Model. Journal of Statistical Theory and Applications, 16, 219-238.

Bashkar, E., Torabi, H., Dolati, A. and Belzunce, F. (2018). f-Majorization with Applications to Stochastic Comparison of Extreme Order Statistics. Journal of Statistical Theory and Applications, 17, 520-536.

Bon, J. L. and Păltănea, E. (1999). Ordering Properties of Convolutions of Exponential Random Variables, Lifetime Data Analysis 5, 185-192.

Fang, L. and Balakrishnan, N., (2016). Likelihood Ratio Order of Parallel Systems with Heterogeneous Weibull Components. Metrika, 79, 693-703.

Fang, R., Li, C. and Li, X., (2015). Stochastic Comparisons on Sample Extremes of Dependent and Heterogenous Observations. Statistics, 1-26.

Fang, L. and Zhang, X., (2013). Stochastic Comparisons of Series Systems with Heterogeneous Weibull Components. Statistics and Probability Letters, 83, 1649-1653.

Fang, L. and Zhang, X., (2015). Stochastic Comparisons of Parallel Systems with Exponentiated Weibull Components. Statistics and Probability Letters, 97, 25-31.

Khaledi, B. E., and Kochar, S. (2006). Weibull Distribution: Some Stochastic Comparisons Results. Journal of Statistical Planning and Inference, 136, 3121-3129.

Kundu, A., and Chowdhury, S. (2016). Ordering Properties of Order Statistics from Heterogeneous Exponentiated Weibull Models. Statistics and Probability Letters, 114, 119-127.

J. Statist. Res. Iran 16 (2019): 101-120 
Kundu, A., Chowdhury, S., Nanda, A. K. and Hazra, N. K. (2016). Some Results on Majorization and Their Applications. Journal of Computational and Applied Mathematics, 301, 161-177.

Li, X., and Fang, R. (2015). Ordering Properties of Order Statistics from Random Variables of Archimedean Copulas with Applications. Journal of Multivariate Analysis, 133, 304-320.

Li, C. and Li, X., (2015). Likelihood Ratio Order of Sample Minimum from Heterogeneous Weibull Random Variables. Statistics and Probability Letters, 97, 46-53.

Li, H. and Li, X., (2013). Stochastic Orders in Reliability and Risk. Springer, New York.

Marshall, A.W., Olkin, I. and Arnold, B.C., (2011). Inequalities: Theory of Majorization and its Applications. Springer, New York.

McNeil, A. J. and Neslehova, J. (2009). Multivariate Archimedean Copulas, d-Monotone Functions and $\ell_{1}$-Norm Symmetric Distributions, The Annals of Statistics, 3059-3097.

Mudholkar, G. S., and Srivastava, D. K. (1993). Exponentiated WeibullFamily for Analyzing Bathtub Failure-rate Data. IEEE Transactions on Reliability, 42, 299-302.

Nelsen, R.B., (2006). An Introduction to Copulas, Springer, New York.

Shaked, M. and Shanthikumar, J.G., (2007). Stochastic Orders, Springer, New York.

Torrado, N. (2015). Comparisons of Smallest Order Statistics from Weibull Distributions with Different Scale and Shape Parameters. Journal of the Korean Statistical Society, 44, 68-76.

Torrado, N., and Kochar, S. C. (2015). Stochastic Order Relations Among Parallel Systems from Weibull Distributions. Journal of Applied Probability, 52, 102-116.

\section{Esmaeil Bashkar}

Department of Statistics,

Velayat University,

Iranshahr, Iran.

email:e.bashkar@velayat.ac.ir 